\title{
Chemical characterization, antioxidant properties and anti-inflammatory activity of Chinese water chestnut extracts
}

\author{
Jinfeng Yang ${ }^{\mathrm{a}}$, Xiaoxian Tang, ${ }^{\mathrm{a}, *}$, Liang Shuai ${ }^{\mathrm{a}}$, Yong Soo Kwon ${ }^{\mathrm{b}}$, Myong Jo Kim ${ }^{\mathrm{c}, *}$ \\ ${ }^{a}$ Research Institute of Food Science \& Engineering Technology, Hezhou University, Hezhou 542899 China \\ b Department of Pharmacy, Kangwon National University, Chuncheon 200-701 Republic of Korea \\ c Bioherb Research Institute, Kangwon National University, Chuncheon 200-701 Republic of Korea
}

*Corresponding authors, e-mail: tangxiaoxian2016@163.com, kimmjo@kangwon.ac.kr

Received 3 Jul 2019

Accepted 28 Mar 2020

\begin{abstract}
Ferulic acid, caffeic acid and p-coumaric acid have been identified in the Eleocharis dulcis (Burm.f.) Trin. ex Hensch. (Chinese water chestnut (CWC)) ethyl acetate extract. The ethyl acetate extract of CWC had the highest DPPH radical scavenging activity $\left(\mathrm{IC}_{50}=12.81 \pm 0.09 \mu \mathrm{g} / \mathrm{ml}\right)$, which was higher than that of the positive control butyl hydroxy anisd $\left(\mathrm{IC}_{50}=14.73 \pm 0.08 \mu \mathrm{g} / \mathrm{ml}\right.$ ). The reducing power of $0.5 \mathrm{mg} / \mathrm{ml}$ CWC was 1.21 at $0.5 \mathrm{mg} / \mathrm{ml}$. The ethyl acetate extract significantly attenuated the production of reactive oxygen species in a dosedependent manner. The anti-inflammatory activity of CWC extracts was assessed in lipopolysaccharide-induced RAW 264.7 macrophages. The highest anti-inflammatory activity was conferred by $300 \mu \mathrm{g} / \mathrm{ml}$ CWC ethyl acetate extract, which also significantly reduced nitric oxide production $(p<0.05)$. Treatment with the CWC ethyl acetate extract downregulated the expression of TNF- $\alpha$, iNOS, and COX-2 genes. For the first time the identification of phenolic acids from CWC and their correlation with anti-inflammatory activity were reported in this work.
\end{abstract}

KEYWORDS: antioxidant, anti-inflammatory, Chinese water chestnut, phenolic acids, peritoneal macrophages

\section{INTRODUCTION}

Biochemical intermediates in the pathways within the human body can react with oxygen to produce highly reactive molecules known as reactive oxygen species (ROS) or free radicals. If the endogenous pool of antioxidants is insufficient to compensate for the rate of ROS production and the activity of ROS, reactive nitrogen species (RNS), and free radicals, the resulting oxidative stress can enhance the pathophysiological mechanisms of several diseases such as inflammatory diseases. Inflammation is a dynamic process that occurs in vascularized tissue in response to stimuli that cause cell injury and tissue degeneration. ROS and RNS have key roles in mediating the development and progression of degenerative tissue processes [1]. Free radicals can induce lipid peroxidation, which has been linked to cancer. The high production of ROS in tumor cells has contributed to the development of sophisticated cellular responses to counterbalance the resulting oxidative stress by rapidly proliferating. Based on these data, drugs with antioxidant activities are widely used for the treatment of many disorders including inflammatory diseases, cancer and anxiety neurosis.

Plants are natural sources of a diverse array of functional metabolites and compounds. Many plant phytochemicals (secondary metabolites) have antioxidant [2], anti-inflammatory [3], anti-cancer and anti-bacterial properties. Chinese water chestnut (CWC, Eleocharis dulcis (Burm.f.) Trin. ex Hensch.) is a hydrophytic vegetable that grows in marshes or ponds. It is widely consumed in China because of its unique taste and high nutrient value. It is valued for its medicinal properties. CWC exhibits strong antimicrobial and antioxidant activities [4], and has been used in traditional Chinese medicine to treat pharyngitis, laryngitis, enteritis, hepatitis and hypertension [5]. To the best of our knowledge, there is little or no information on the medicinal properties of CWC extracts, which could include valuable antioxidant and anti-inflammation compounds. In this study, we investigate the medicinal properties of CWC extracts, focusing on antioxidant properties, 2,2-diphenyl-1-picrylhydrazyl (DPPH)-radical scavenging activity, reducing power, total phenolic and flavonoid contents, and antiinflammatory activity in RAW 264.7 macrophage 
cells in vitro. And the main antioxidants from CWC were isolated, identified and quantified.

\section{MATERIALS AND METHODS}

\section{Samples preparation}

The whole plant of Chinese water chestnut was obtained from Hezhou, Guangxi, China in December 2017. The sample was extracted with $100 \%$ methanol. The crude extract was suspended in deionized water and partitioned sequentially with n-hexane, ethyl-acetate (EtOAc) and butanol (water saturated $\mathrm{BuOH}$ ) fractions for further analysis.

\section{Isolation of biological compounds}

In total, three compounds were isolated following the procedure described below. The EtOAc fraction $(25.6 \mathrm{~g})$ was separated on ion exchange chromatography using stepwise gradient elution with methanol:water (50:50-100:0) to yield 4 fractions (Fr. 1-Fr. 4). Fraction Fr. 1 $(10.5 \mathrm{~g})$ was chromatographed on silica gel (141.4 g, 0.3-0.2 mm) by gradient elution with dichloromethane:methanol (100:0-0:100) to yield 10 subfractions (Fr. 1-1-Fr. 1-10). Subfraction Fr. 1-8 (2.1 g) chromatographed on an octadecylsi-

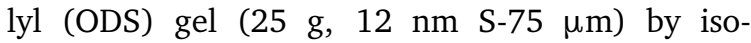
cratic elution with methanol to give compound 1 $(15.6 \mathrm{mg})$ and compound $2(28.7 \mathrm{mg})$. Fraction Fr. 3 (8.4 g) was chromatographed on silica gel (90.4 g, $0.3-0.2 \mathrm{~mm}$ ) by gradient elution with chloroform:methanol (50:0-0:50) then separated by Sephadex LH-20 gel by isocratic elution with methanol:water (7:3) to give compound 3 . The three compounds were identified by ${ }^{1} \mathrm{H}$ and ${ }^{13} \mathrm{C}$ NMR analyses.

\section{DPPH radical scavenging capacity}

The DPPH radical scavenging activities of the extracts were determined according to the method of Blois [6] and expressed as the inhibition percentage of free radicals calculated as: $(\%)$ inhibition $=(1-$ absorbance of sample/absorbance of control) $\times 100$. The $\mathrm{IC}_{50}$ value $(\mu \mathrm{g} / \mathrm{ml})$ represents the concentration at which the scavenging activity is $50 \%$.

\section{Reducing power assay}

The reducing power of the extracts was measured by the method of Oyaizu [7]. Extract or fraction was mixed with $\mathrm{Na}_{3} \mathrm{PO}_{4}$ buffer and potassium ferricyanide, after $20 \mathrm{~min}$, trichloroacetic acid was added and the absorbance was measured at $700 \mathrm{~nm}$.

\section{Determination of total phenolic and flavonoid content}

Total phenolic content was estimated by the FolinCiocalteu method [8]. The data were expressed as gallic acid equivalent (GAE) per mg of extract, based on the calibration curve of gallic acid. The total flavonoid content was determined by Park [9]. The data were expressed as $\mathrm{mg} / \mathrm{g}$ quercetin.

\section{Assay of ROS production}

The evolution of reactive oxygen species (ROS) using dichlorofluorescin diacetate (DCFH-DA) assay [10]. ROS levels were measured by flow cytometry.

\section{Nitric oxide (NO) production}

RAW 264.7 cells were plated in 6-well plates and stimulated with lipopolysaccharide (LPS) in the absence or presence of various concentrations of CWC. The procedure for NO concentration was measured using the Griess reaction. The absorbance was measured at $540 \mathrm{~nm}$.

\section{Preparation of total RNA and cDNA synthesis}

Total RNA and PCR analyses were described previously [11]. The primer sequences were as follows:

$\beta$-actin-F, $5^{\prime}$-TAC AGC TTC ACC ACC ACA GC- $3^{\prime}$, $\beta$-actin-R, $5^{\prime}$-AAG GAA GGC TGG AAA GC- $3^{\prime}$, iNOS-F, $5^{\prime}$-TTT CCA GAA GCA GAA TGT GA- $3^{\prime}$, iNOS-R, 5' -AAC ACC ACT TTC ACC AAG AC-3', COX-2-F, 5'-TGA GTA CCG CAA ACG CTT CTC-3', COX-2-R, 5'-TGG ACG AGG TTT TTC CAC CAG-3', TNF- $\alpha$-F, $5^{\prime}$-CTA CTC CCA GGT TCT CTT CAA TG-3', TNF- $\alpha$-R, $5^{\prime}$ - GCA GAG AGG AGG TTG ACT TTC- $3^{\prime}$.

\section{Statistical Analysis}

Data were statistically evaluated via Duncan's oneway analysis of variance (ANOVA). The level is considered significant when $p<0.05$. Data were presented as means $\pm \mathrm{SD}$.

\section{RESULTS AND DISCUSSION}

\section{Determination of antioxidant activity in CWC extracts}

We investigated the antioxidant activity of CWC extracts by measuring total phenolic and flavonoid content, reducing power, DPPH-radical scavenging activity, and attenuation of ROS generation. We 
Table 1 Total phenolic and flavonoid contents and DPPH radical scavenging activity of CWC extract and fractions.

\begin{tabular}{lccr}
\hline Fraction & $\begin{array}{c}\text { DPPH radical } \\
\text { scavenging activity } \\
\text { IC } 50(\mu \mathrm{g} / \mathrm{ml})\end{array}$ & $\begin{array}{c}\mathrm{TPC}^{1} \\
(\mathrm{mg} \mathrm{GAE} / \mathrm{g})\end{array}$ & $\begin{array}{c}\mathrm{TFC}^{2} \\
(\mathrm{mg} \mathrm{QE} / \mathrm{g})\end{array}$ \\
\hline $\mathrm{M}$ & $43.59 \pm 0.35^{\mathrm{b}}$ & $106.27 \pm 4.96^{\mathrm{b}}$ & $35.29 \pm 0.64^{\mathrm{b}}$ \\
$\mathrm{H}$ & $317.34 \pm 0.28^{\mathrm{d}}$ & $6.50 \pm 1.00^{\mathrm{d}}$ & $0.45 \pm 2.27^{\mathrm{d}}$ \\
$\mathrm{E}$ & $12.81 \pm 0.09^{\mathrm{a}}$ & $271.91 \pm 1.67^{\mathrm{a}}$ & $182.21 \pm 2.86^{\mathrm{a}}$ \\
$\mathrm{B}$ & $281.46 \pm 0.05^{\mathrm{c}}$ & $20.34 \pm 1.92^{\mathrm{c}}$ & $15.59 \pm 1.22^{\mathrm{c}}$ \\
$\mathrm{W}$ & - & - & - \\
$\mathrm{BHA}$ & $14.73 \pm 0.08$ & & \\
\hline
\end{tabular}

${ }^{1}$ Total phenolic contents; ${ }^{2}$ total flavonoid contents; $\mathrm{M}$, methanol extract; $\mathrm{H}$, n-hexane fraction; $\mathrm{E}$, ethyl acetate fraction; $\mathrm{B}$, butanol fraction; $\mathrm{W}$, water fraction.

"Values within the same column followed by different letters (a-e) are significantly different at $p<0.05$.

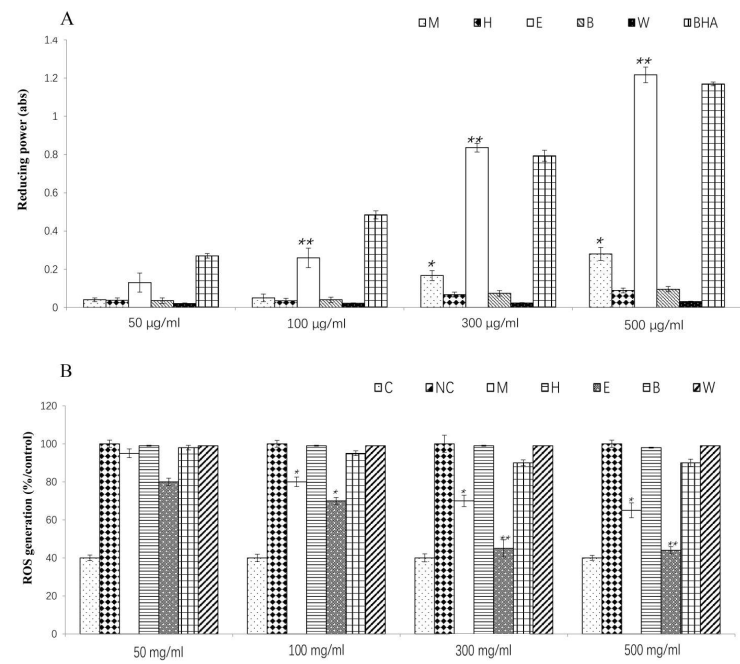

Fig. 1 Antioxidant activity of the CWC extract and fractions. (A) Reducing power of the CWC. (B) Reactive oxygen species (ROS) generation in lipopolysaccharideinduced RAW 264.7 cells following treatment with CWC. $\mathrm{M}$, methanol extract; $\mathrm{H}$, n-hexane fraction; $\mathrm{E}$, ethyl acetate fraction; B, butanol fraction; W, water fraction. Values represent the mean \pm SD. Significantly different at $* p<0.05$ and $* * p<0.01$.

found that CWC extract contained significant antioxidant activity. The highest DPPH-radical scavenging activity was contained in the ethyl acetate extract $\left(\mathrm{IC}_{50}=12.81 \pm 0.09 \mu \mathrm{g} / \mathrm{ml}\right)$, which had higher activity than other extracts (n-hexane, butanol and water), and was superior to the positive control butyl hydroxy anisd (BHA, $\mathrm{IC}_{50}=$ $14.73 \pm 0.08 \mu \mathrm{g} / \mathrm{ml}$ ) (Table 1). The ethyl acetate extract at $500 \mu \mathrm{g} / \mathrm{ml}\left(\mathrm{OD}_{700}=1.21\right)$ had the highest reducing power of all extracts (Fig. 1A). Consensus opinion considers that the antioxidant activity in plant extracts is related to the total phenolic content (TPC) and total flavonoid content (TFC). This correlates with our results showing that TPC was isolated in the ethyl acetate extract $(271.91 \pm 1.67 \mathrm{mg}$ gallic acid equivalent (GAE)/g) and was greater than that in the methanol extract $(106.27 \pm 4.69 \mathrm{mg} \mathrm{GAE} / \mathrm{g})$ or other extracts $(>\mathrm{BuOH}$ extract $(20.34 \pm 1.92$ $\mathrm{mg} \mathrm{GAE} / \mathrm{g})$ and $>$ n-hexane extract $(6.50 \pm 1.00$ mg GAE/g)). The ethyl acetate extract displayed higher TFC $(128.21 \pm 2.86 \mathrm{QE} / \mathrm{g})$ than other extracts (Table 1).

ROS are associated with many forms of apoptosis and can act as secondary messengers that activate the production of mediators involved in inflammatory processes. Therefore, we determined whether CWC extracts affected ROS generation. The ethyl acetate extract significantly reduced ROS production in a dose-dependent manner (Fig. 1B). A concentration of $300 \mu \mathrm{g} / \mathrm{ml}$ ethyl acetate significantly inhibited ROS production to $44.78 \%$ of that produced in non-treated LPS-induced cells. These results suggest that the ethyl acetate extract can inhibit ROS production by scavenging free radical. These effects were in agreement with a report by Ghosh [12], who suggest that Aronia melanocarpa 'Viking' extracts displayed strong radical scavenging activity and may have a crucial role in inhibiting ROS generation. Oxidative stress has been proposed as a causative factor in the toxicity of numerous chemicals, and it has a significant role in the pathogenesis of several human diseases including inflammatory conditions, certain cancers and cataracts. Antioxidants prevent the adverse effects of oxygen reactivity by capturing and neutralizing free radicals, thereby eliminating free radical damage to the human body.

\section{Effect of CWC extracts on nitric oxide production}

To examine whether the extracts attenuated the LPS-induced inflammatory response, RAW 264.7 cells were treated with different doses of CWC extract plus LPS. After $24 \mathrm{~h}$, the LPS-induced inflammatory response was evident compared with controls. The CWC ethyl acetate extract significantly inhibited NO production in dose-dependent manner (Fig. 2A). Treatment of RAW 264.7 cells with $300 \mu \mathrm{g} / \mathrm{ml}$ ethyl acetate extract reduced NO production by approximately $60 \%$ compared with control. High concentrations of the CWC methanol extract slightly induced NO production, whereas the n-hexane, butanol and water extracts did not significantly affect NO production. These results 
A
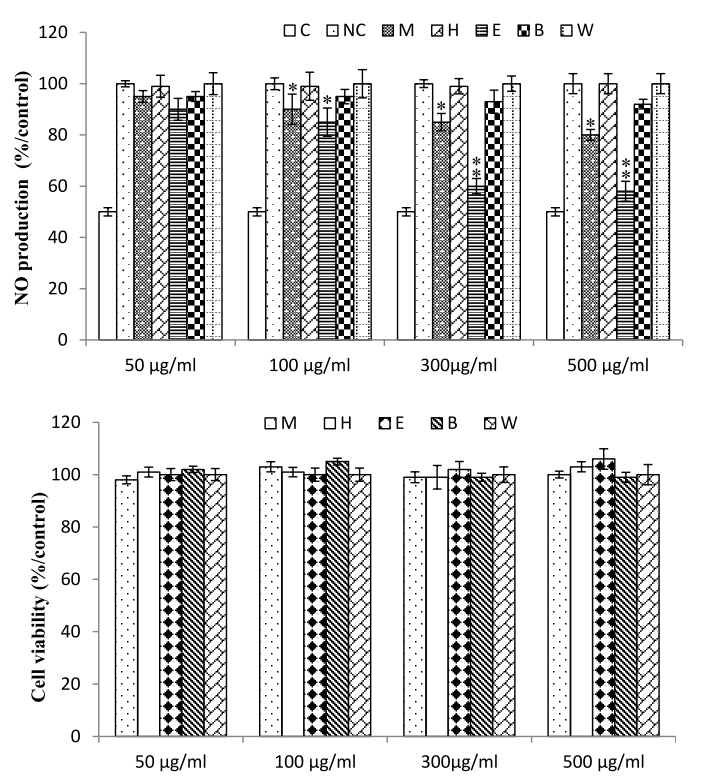

B

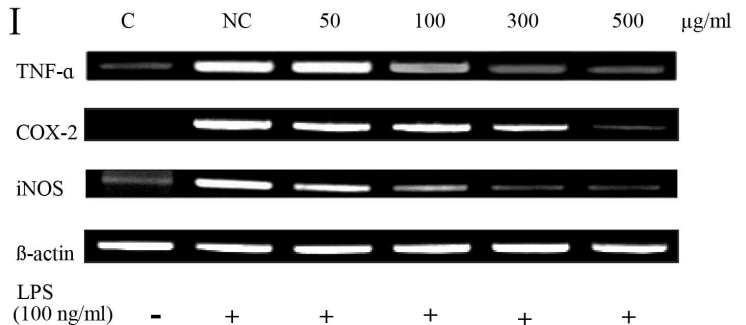

II

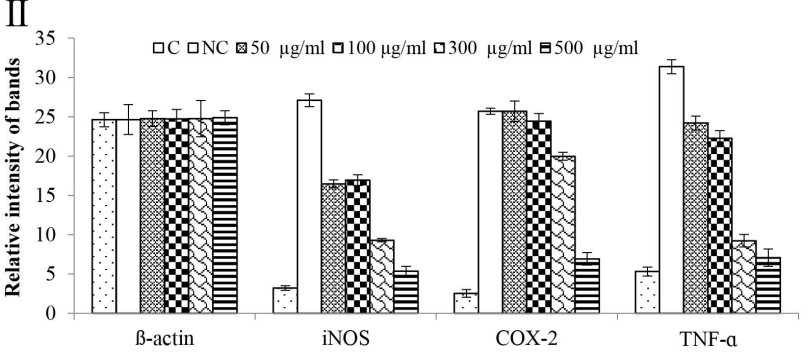

Fig. 2 The anti-inflammatory effects of CWC. (A) The effect of CWC extract on the production of NO in RAW 264.7 cells. Values represent the mean \pm SD. Significantly different at $* p<0.05$ and $* * p<0.01$. (B) The effect of EtOAc fraction from CWC on LPS-induced $\beta$-actin, iNOS, COX-2 and TNF- $\alpha$ expression in RAW 264.7 cells. (I) Gel electrophoresis of amplified product of $\beta$-actin, iNOS, COX-2 and TNF- $\alpha$; (II) quantitative data of panel (B); C, control; NC, negative control.

correlated well with the antioxidant results, indicating that the ethyl acetate extract contained high phenolic content and substantial antioxidant activity. Antioxidants act by scavenging free radicals such as ROS, NO and hydroxyl radicals. The strong scavenging activity against ROS, which has an important role in the pathogenesis of inflammation, along with reduced NO production, explains in part the beneficial effects of CWC extracts in the treatment of inflammation. We propose that the radical-scavenging activity and anti-inflammatory activity of phenolic compounds are inseparably connected. Other studies also have reported that phenolic compounds significantly inhibit pro-inflammatory enzymes and cytokine-induced inflammatory responses in human and mouse cells via their potent antioxidant activities [13]. Inhibition of NO production may contribute to the anti-inflammatory activity of the CWC ethyl acetate extract, so it was selected for use in subsequent experiments.

\section{Effect of the CWC ethyl acetate extract}

Nitric oxide acts as a pro-inflammatory agent in inflammation. NO is synthesized by inducible nitric oxide synthase (iNOS) in response to proinflammatory agents such as lipopolysaccharides (LPS) [14].
LPS can trigger inflammation and induce the overexpression of many inflammatory mediators. Antiinflammatory agents act by modulating the activities of pro-inflammatory enzymes and cytokines [13]. Our previous study revealed that the ethyl acetate extract of CWC significantly inhibited NO generation in a dose-dependent manner. Therefore, we investigated whether the ethyl acetate extract could inhibit the expression of proinflammatory cytokine genes such as TNF- $\alpha, C O X-2$ and iNOS in RAW 264.7 cells. LPS dramatically up-regulated TNF- $\alpha$ mRNA expression (Fig. 2B). By contrast, the ethyl acetate extract inhibited TNF- $\alpha$ mRNA expression in LPS-induced RAW 264.7 cells. Similarly, iNOS and COX-2 expression was markedly stimulated by LPS, whereas these expression levels declined in a dose-dependent manner after treatment with the ethyl acetate extract. Treatment with $500 \mu \mathrm{g} / \mathrm{ml}$ ethyl acetate extract reduced $i N O S, C O X-2$ and TNF$\alpha$ expression to almost undetectable levels. COX2 is an inducible gene that converts arachidonic acid into prostaglandins, and it is implicated in pathological conditions such as inflammation, cancer and Alzheimer's disease [15]. The production of TNF- $\alpha$ also is crucial for the immune response to many inflammatory stimuli. For example, TNF- $\alpha$ 


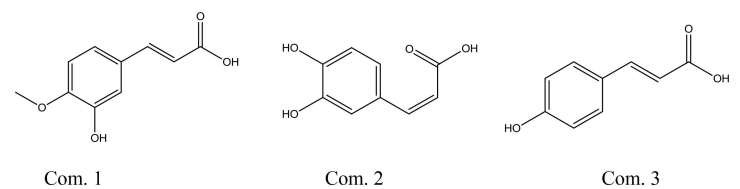

Fig. 3 Chemical structures of the compounds isolated from Chinese water chestnut.

is overexpressed in acute and chronic inflammatory disorders [13]. These combined results indicate that CWC contains anti-inflammatory activity that can reduce the expression of pro-inflammatory signals and inhibit the production of inflammatory cytokines.

\section{Identification of the chemical structures of the isolated compounds}

The following compounds were identified by comparison of its ${ }^{1} \mathrm{H}$ and ${ }^{13} \mathrm{C}$ NMR data with literature and are in agreement with the proposed structures. The structures of compounds 1-3 are presented in Fig. 3. Compound 1 was identified as ferulic acid, by NMR analysis, and comparison with its literature data [16]. Compounds 2 and 3 were identified as caffeic acid and p-coumaric acid, respectively, by NMR analysis, and comparison with their literature data [17].

\section{Antioxidant capacities of compounds}

The compounds isolated from CWC were examined for their inhibitory activity against the DPPH radical. Compound 2 (at a concentration of $13.27 \pm 0.33 \mu \mathrm{mol} / \mathrm{ml}$ ) showed higher radical scavenging activity than compound $1\left(\mathrm{IC}_{50}=\right.$ $40.36 \pm 0.98 \mu \mathrm{mol} / \mathrm{ml})$. Compound 3 possessed weak activity $\left(\mathrm{IC}_{50}=2307.15 \pm 1.21 \mu \mathrm{mol} / \mathrm{ml}\right)$. ROS production can be inhibited by antioxidants capable of entering live cells and can be further inhibited by anti-inflammatory compounds in a complex product. The pretreatment of RAW 264.7 cells with samples of compounds 1,2 and 3 resulted in inhibition of the formation of ROS by the RAW 264.7 cells (Fig. 4). Untreated RAW 264.7 cells (no sample, no LPS) served as a baseline, and RAW 264.7 cells treated with LPS in the absence of sample served to show maximum ROS production. RAW 264.7 cells treated with the sample produced fewer ROS than cells treated with LPS in the absence of sample. The inhibition of ROS formation was dosedependent and, at the highest dose of compound 2 tested $(100 \mu \mathrm{mol} / \mathrm{ml})$, the inhibition was highly
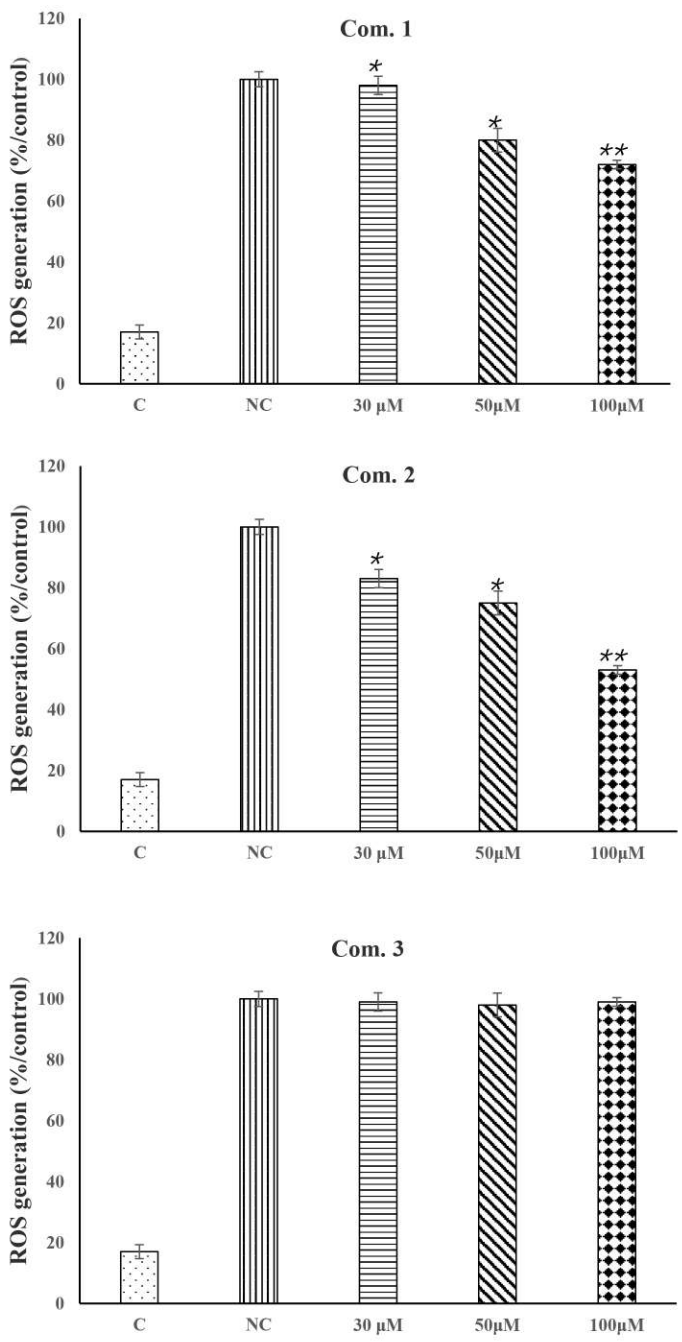

Fig. 4 Antioxidant activity of compounds from CWC. Reactive oxygen species (ROS) generation in lipopolysaccharide-induced RAW 264.7 cells following treatment with compounds from CWC.

significant. These results confirm that compounds 1 and 2 scavenged free radicals and inhibited radicalmediated oxidation in RAW 264.7 cells.

\section{CONCLUSION}

This study investigated the antioxidant and antiinflammatory activities of Chinese water chestnut (CWC) extract using the DPPH assay to test for radical scavenging activity and RT-PCR analysis of proinflammatory signal expression in LPS-stimulated RAW 264.7 cells, respectively. The results showed that CWC extract contained high DPPH radical scavenging activity and high phenolic and flavonoid 
contents. CWC extract significantly reduced ROS production in a dose-dependent manner. Our results clearly demonstrate that CWC extract inhibited NO production and down regulated $\mathrm{NOS}, \mathrm{COX}-2$ and $T N F-\alpha$ expression. In addition, we isolated ferulic acid (compound 1), caffeic acid (compound 2) and p-coumaric acid (compound 3) from the ethyl acetate fraction of the CWC and evaluated for the antioxidant properties. These data indicate that CWC could be developed as a herbal remedy for preventing and treating inflammatory diseases.

Acknowledgements: This research was supported by the research grant from Bioherb Research Institute, Kangwon National University, the Natural Science Foundation of Guangxi province (No. 2019GXNSFBA245040), the doctor's scientific research foundation of Hezhou University (HZUBS201612). Special fund for distinguished experts in Guangxi.

\section{REFERENCES}

1. Shah MD, D'Souza UJA, Iqbal M (2017) The potential protective effect of Commelina nudiflora $\mathrm{L}$. against carbon tetrachloride $\left(\mathrm{CCl}_{4}\right)$-induced hepatotoxicity in rats, mediated by suppression of oxidative stress and inflammation. Environ Health Prev Med 22, ID 66.

2. Qadir A, Ali A, Arif M, Al-Rohaimi AH, Singh SP, Ahmad U, Khalid M, Kumar A (2018) Solvent extraction and GC-MS analysis of sesame seeds for determination of bioactive antioxidant fatty acid/fatty oil components. Drug Res (Stuttg) 68, 344-348.

3. Ghimeray AK, Lee HY, Kim YH, Ryu EK, Chang MS (2015) Evaluation of antioxidant and antiInflammatory effect of Rhododendron brachycarpum extract used in skin care product by in vitro and in vivo test. Tech Invest 6, 105-111.

4. Li XR, Luo YH, He J, Peng LY, Wu XD, Du RN (2013) Phenolic constituents and antioxidant activity of Eleocharis tuberosa peels. Nat Prod Res Dev 25, 1615-1620.

5. Liu X, Zhao L, Zhou A (2006) Preliminary study on functional component and functional activities of waste slurry derived in processing water chestnut starch. Food Sci 27, 251-256.
6. Blois MS (1958) Antioxidant determinations by the use of a stable free radical. Nature 181, 1199-1200.

7. Oyaizu M (1986) Studies on products of browning reactions: antioxidative activities of products of browning reaction prepared from glucosamine. Jpn $J$ Nutr Diet 44, 307-315.

8. Singleton VL, Rossi J (1965) Colorimetry of total phenolics with phosphomolybdic-phosphotungstic acid reagents. Am J Enol Viticult 16, 144-158.

9. Park YK, Koo MH, Ikegaki M, Contado JL (1997) Comparison of the flavonoid aglycone contents of Apis mellifera propolis from various regions of Brazil. Arquivos Biol Tecnol 40, 97-106.

10. Wang H, Joseph JA (1999) Quantifying cellular oxidative stress by dichlorofluorescein assay using microplate reader. Free Radical Bio Med 27, 612-616.

11. Murakami Y, Ishii H, Takada N, Tanaka S, Machino M, Ito S, Fujisawa S (2008) Comparative antiinflammatory activities of curcumin and tetrahydrocurcumin based on the phenolic $\mathrm{O}-\mathrm{H}$ bond dissociation enthalpy, ionization potential and quantum chemical descriptor. Anticancer Res 28, 699-707.

12. Ghosh M, Kim IS, Lee YM, Hong SM, Lee TH, Lim JH, Debnath T, Lim BO (2018) The effects of Aronia melanocarpa 'Viking' extracts in attenuating RANKL-induced osteoclastic differentiation by inhibiting ROS generation and c-FOS/NFATc1 signaling. Molecules 23, ID 615.

13. Aro AO, Dzoyem JP, Eloff JN, McGaw LJ (2016) Extracts of six Rubiaceae species combined with rifampicin have good in vitro synergistic antimycobacterial activity and good anti-inflammatory and antioxidant activities. BMC Complement Altern Med 16, ID 385.

14. Lu YC, Yeh WC, Ohashi PS (2008) LPS/TLR4 signal transduction pathway. Cytokine 42, 145-151.

15. Jeon YJ, Kim BH, Kim S, Oh I, Lee S, Shin J, Kim TY (2013) Rhododendrin ameliorates skin inflammation through inhibition of NF- $\kappa \mathrm{B}, \mathrm{MAPK}$, and PI3K/Akt signaling. Eur. J. Pharmacol 714, 7-14.

16. Prachayasittikul S, Suphapong S, Worachartcheewan A, Lawung R, Ruchirawat S, Prachayasittikul V (2009) Bioactive metabolites from Spilanthes acmella Murr. Molecules 14, 850-867.

17. Samy MN, Sugimoto S, Matsunami K (2014) Bioactive compounds from the leaves of Eugenia uniflora. Nat Prod 7, 37-47. 